\title{
Identification of NR5A1 (SF-1/AD4BP) gene expression modulators by large-scale gain and loss of function studies
}

\author{
Noriko Sakai*, Hiromi Terami*, Shinobu Suzuki, Megumi Haga, Ken Nomoto, Nobuko Tsuchida, \\ Ken-ichirou Morohashi ${ }^{1}$, Naoaki Saito ${ }^{2}$, Maki Asada ${ }^{3}$, Megumi Hashimoto ${ }^{3}$, Daisuke Harada ${ }^{3}$, \\ Hiroshi Asahara ${ }^{3}$, Tetsuya Ishikawa, Fumiki Shimada and Kazuhiro Sakurada
}

Department of Stem Cell Based Drug Discovery, Research Center Kobe, Bayer Yakuhin Ltd, Kobe, Hyogo 650-0047, Japan

${ }^{1}$ Division of Sex Differentiation, National Institute for Basic Biology, Myodaiji-cho, Okazaki, Nagoya 444-8787, Japan

${ }^{2}$ Laboratory of Molecular Pharmacology, Biosignal Research Center, Kobe University, Kobe, Hyogo 657-8501, Japan

${ }^{3}$ Department of Systems Biomedicine, National Research Institute for Child Health and Development, 157-8535 Tokyo, Japan

(Correspondence should be addressed to K Sakurada; Email: kazuhiro.sakurada@gmail.com)

*(N Sakai and H Terami contributed equally to this work)

\begin{abstract}
Nuclear receptor subfamily 5 , group A, member 1 (NR5A1 previously known as $S F-1 / A D 4 B P)$ is a transcription factor involved in the development of adrenal/gonadal tissues and steroidogenic linage cell differentiation in adult somatic stem cells. To understand the cellular signaling network that regulates NR5A1 gene expression, loss of function screening with an siRNA kinome library, and gain of function screening with an addressable full-length cDNA library representing one quarter of the human genome was carried out. The NR5A1 gene expression was activated in mesenchymal stem cells by siRNA directed against protein kinase $\mathrm{C}(\mathrm{PKC})-\delta$, erb-B3, RhoGAP (ARHGAP26), and hexokinase 2 , none of which were previously known to be
\end{abstract}

involved in the NR5A1 gene expression. Among these, we identified crosstalk between erb-B3 and $\mathrm{PKC}-\delta$ signaling cascades. In addition, the gain of function studies indicated that sex-determining region Y (SRY)-box 15 (SOX15), TEA domain family member 4, KIAA1257 (a gene of unknown function), ADAM metallopeptidase with thrombospondin type 1 motif 6 , Josephin domain containing 1 , centromere protein, TATA box-binding protein-associated factor 5-like RNA polymerase, and inducible T-cell co-stimulator activate NR5A1 gene expression. These results provide new insights into the molecular mechanisms of NR5A1 gene expression.

Journal of Endocrinology (2008) 198, 489-497

\section{Introduction}

Ovarian folliculogenesis has an essential role in oocyte maturation and steroid production (Palter et al. 2001). The wall of a mature preovulatory follicle consists of granulosa, theca interna, and theca externa cells that produce three classes of steroid hormones, progestins, androgens, and estrogens (Vanderhyden 2002). Theca interna cells produce androgens, whereas granulosa cells convert androgens to estrogens. Progesterone is the major product of granulosa cells (Vanderhyden 2002). The human follicle pool decreases significantly with age and the number of follicles is reduced to almost zero at menopause (Broekmans et al. 2007). The decline in estrogen at menopause may cause symptoms such as dizziness and depression, and post-menopausal women are at risk of developing osteoporosis (Broekmans et al. 2007). Hormone replacement is effective for menopause disorders; however, large-scale clinical studies demonstrated that estrogen treatment can be a risk factor for myocardial infarction, stroke, breast cancer, and uterine cancer (Rossouw et al. 2002). At present, the
FDA (Food and Drug Administration) recommends using the lowest dose of hormone replacement therapy (HRT) for the shortest duration (Cirigliano 2007). One of the major problems of estrogen replacement therapy is the optimization of the dose of estrogen in each individual patient (Cirigliano 2007). Production of steroids in the ovary is tightly regulated by the hypothalamus-anterior pituitary axis. Androgen production from theca interna cells is stimulated by luteinizing hormone (LH; Magoffin 2005). Follicle-stimulating hormone (FSH) stimulates the conversion of androgen to estrogen in granulosa cells by inducing aromatase, which catalyzes estrogen biosynthesis from C19 steroids (Simpson \& Davis 2001). On the other hand, estrogen negatively regulates the production of LH and FSH in the anterior pituitary (de Ziegler et al. 2007). Thus, a treatment that can regenerate this hormone loop might have advantages compared with standard HRT, as it should result in tight control of hormone levels in blood by the body's natural feedback loops.

One possible therapeutic strategy to achieve this is to develop transplantable estrogen-producing cells in vitro. 
To achieve this, nuclear receptor subfamily 5, group A, member 1 (NF5A1 that was previously known as $S F-1$ or $A D 4 B P)$ is a key molecule. NF5A1 is the steroidogenic tissue-specific transcription factor that controls the expression of the steroidogenic cytochrome P450 genes in endocrine tissues, such as the adrenal cortex, testis, and ovary (Morohashi et al. 1992). Previously, Crawford et al. (1997) using stable ectopic expression of NF5A1 in mouse embryonic stem (ES) cells, showed that NF5A1 regulates differentiation and promotes steroidogenesis in ES cells, demonstrating that $N F 5 A 1$ is a dominant regulator of the steroidogenic cell phenotype. Also, it has been reported that primary long-term-cultured mesenchymal stem cells (MSCs) infected with an adenovirus-encoding bovine SF-1 (bSF-1) synthesized de novo multiple steroid hormones (Gondo et al. 2004, Tanaka et al. 2007). This has been confirmed by Yazawa et al. (2006) who established steroidogenic cells by NF5A1 forced expression.

Another therapeutic strategy is to develop pharmacological interventions to activate endogenous steroidogenesis in the ovary. Primordial follicles appear to contain only granulosa cells. It is well known that theca cells differentiate from a population of unspecialized MSCs in the ovarian stroma (Magoffin 2005). Consistent with these findings, theca stem cells, which appear similar to fibroblasts and MSCs, were isolated recently (Honda et al. 2007). The presence of theca stem cells/ovarian MSCs in post-menopausal women has not been shown. However, it is likely that ovarian MSCs share common features with MSCs in other tissues. Thus, the therapeutic strategy for pharmacological interventions is to develop drugs that can stimulate the differentiation of endogenous theca/ ovarian MSCs into estrogen-producing cells.

Adipocytes, which differentiate from MSCs, are known to produce estrogen in an LH/FSH-independent manner. Although the expression of estrogen biosynthesis enzymes is regulated by $N F 5 A 1$ in the ovary, a different transcription factor (NF5A2 previously known as $L R H-1$ ) regulates the expression of aromatase in adipocytes (Clyne et al. 2002). NF5A2 also regulates aromatase expression in the ovary (Saxena et al. 2007); however, NF5A1 is not expressed in adipocytes. Thus, we have focused our studies on the identification of NF5A1 gene expression modulators in MSCs.

To date, only limited factors have been identified that regulate NF5A1 gene expression (Scherrer et al. 2002). However, as an siRNA kinome library has been shown to be useful to identify new regulators of the target of the interest (Chan et al. 2007, Tyner et al. 2008), to identify these modulator genes, we carried out large-scale screening to clarify the cellular signaling network regulating $N F 5 A 1$ gene expression in a comprehensive manner, by loss of function screening with an siRNA kinome library in MSCs. Moreover, gain of function screening with an addressable fulllength cDNA library representing approximately one quarter of the human genome were also carried out using an NF5A1 promoter reporter system in human embryonic kidney (HEK)293 cells.

\section{Materials and Methods}

\section{Cell culture}

Two lines of HEK293, HEK293 FT cells and HEK293 low cells, were obtained from Invitrogen and Microbix (Ontario, Canada) respectively. HEK293 FT cells were established from a fast-growing variant of the 293 cell line that stably expresses the SV40 large T antigen and these cells were mainly used in our study. HEK293 low cells were used when lower background of reporter gene expression is required in the confirmation study, due to its lower transfection efficiency compared with HEK293 FT cells. HEK293 were maintained in Iscove's modified Dulbecco's medium (IMDM; supplemented with 10\% fetal bovine serum (FBS); Sigma-Aldrich). Human bone marrow-derived MSCs (lot number 4F1560; Lonza, Basel, Switzerland) were cultured in MSC medium (MSCGM; Lonza). When the cultures reached subconfluence, the cells were harvested with $0.05 \%$ trypsin and $1 \mathrm{mM}$ EDTA and were replated at $5 \times 10^{3}$ cells per $\mathrm{cm}^{2}$.

\section{siRNAs, $c D N A s$, and reagents}

Both siRNA kinome library and validated siRNAs for hit molecules were purchased from Qiagen. The human cDNA library Mammalian gene collection (MGC), consisting of 6344 clones, was purchased from ATCC. IMAGE clones for protein kinase $\mathrm{C}(\mathrm{PKC})-\delta$, sex-determining region $\mathrm{Y}(\mathrm{SRY})-$ box 15 (SOX15), TEA domain family member 4 (TEAD-4), KIAA1257, ADAM metallopeptidase, Josephin domain containing 1 (JOSD1), centromere protein (CENP), TATA box-binding protein-associated factor 5-like RNA polymerase (TAF5)-like RNA polymerase, and inducible T-cell co-stimulator (ICOS) were all purchased from Open BioSystems. Rottelerin (R5648) was purchased from Sigma.

\section{Plasmids}

bNF5A1 (bSF-1) cDNA was cloned into both pMXs (kindly donated by Dr Kitamura, University of Tokyo, Japan) and pcDNA3.1 vector. The promoter region of human NF5A1 sequence $(5 \mathrm{kbp})$ was digested (BamHI and NotI) from human BAC clone 10J18 (Invitrogen) and was ligated with 77 mer double stranded oligo DNAs $\left(5^{\prime}\right.$-GGC GGC CGC GGG GAC CCC AGG CTG CCG GTC TCC GCC GGC CCT CCC TGA CCC GCT GTC CCT CCG CAG GCG GAC GCC GC-3'). Then the DNA fragment was inserted into a pGL4.20 luciferase expression vector (Promega), named hSF-1 promoter-Luc. A $6.5 \mathrm{kbp}$ DNA fragment for SF-1 promoter-luciferase was digested from hSF-1 promoterLuc and ligated to pMXs-puro-SIN (self inactivating) vectors in reverse orientation, named $\mathrm{pMXs-SIN-hSF-1} \mathrm{promoter-}$ Luc. For creating the human aromatase promoter II reporter construct, $517 \mathrm{bp}$ promoter II region of the aromatase gene, upstream part of exon 2, was cloned from human genomic DNA by PCR with primers $5^{\prime}$-CTC GAG GAT CCT TTG 
AAT TCA ATA GAC AAA C- $3^{\prime}$ and $5^{\prime}$-GAA GCA ACA GGA GCT ATG GTA CCA AGC- $3^{\prime}$. The fragment was cloned into pGL4.20 vector, named hARO promoter-Luc. A DNA fragment for aromatase promoter-luciferase was digested from hARO promoter-Luc, and ligated to pMXspuro-SIN vectors in reverse orientation, named pMXs-SINhARO promoter-Luc.

\section{Adenovirus vectors}

Adenovirus vectors carrying bSF-1 gene (Ad-bSF-1 ) or human SF-1 promoter luciferase (Ad-hSF-1 promoter-Luc) were prepared using Adeno-X Expression System (Clontech). EGFP-expressing adenovirus vector (Ad-EGFP) was purchased from Cosmo Bio. Co. Ltd (Tokyo, Japan).

\section{Detection of hormone production from cultured MSCs}

Prior to the start of stimulation, the MSCs were seeded at $5 \times 10^{3}$ cells/well in 96-well plates, then cells were infected with Ad-bSF-1 or control Ad-EGFP at multiplicity of infection $(\mathrm{MOI})=2$. One day after infection, the medium was replaced with Phenol red-free Dulbecco's modified Eagle's medium (Sigma) supplemented with 10\% charcoaltreated FBS, and cells were cultured with or without $0.5 \mathrm{mM}$ cAMP. Seven days post-infection, supernatants were collected and 17ß-estradiol, testosterone, dehydroepiandrosterone (DHEA), and progesterone concentrations measured with luminescence immunoassay kits (Immuno Biological Laboratories) following the manufacturer's procedure using an EnVision Multilabel Reader (2103; Perkin-Elmer).

\section{Screening of human cDNA library}

From the human cDNA library (MGC collection), $25 \mathrm{ng}$ of each plasmid clone were spotted onto one well of a 384-well plate. hSF-1 promoter-Luc was transfected into 1500 HEK293 cells in each well containing the cDNA library array by using FuGENE HD transfection reagent (Roche). After 3 days, the luciferase activity was measured by SteadyGlo (Promega) using an Arvo Multilabel Reader (1420; Perkin-Elmer).

For the confirmation study with HEK293 cells, cells were grown in 96-well plates at an initial seeding density of $1 \times 10^{4}$ cells/well and cultured for $24 \mathrm{~h}$. Here, we used pMXs-SINhSF-1 promoter-Luc, which had a lowest base background luciferase activity among the promoter-reporter constructs we created, and showed clear response to stimuli such as cAMP, instead of using hSF-1 promoter-Luc. We used 25-80 ng pMXs-SIN-hSF-1 promoter-Luc and 10-16 ng target cDNAs, and they were co-transfected and cells were analyzed for luciferase expression using Steady-Glo by EnVision Multilabel Reader (2103; Perkin-Elmer) after 4 days.

For the confirmation study with the MSCs, $500 \mathrm{ng}$ of the targeted cDNA and $200 \mathrm{ng}$ reporter construct were introduced into $5 \times 10^{4}$ MSCs by electroporation using a
MicroPorator MP-100 (Digital Bio; pulse voltage 990, pulse width 40 , pulse number 1). Cells were transferred to $0.5 \mathrm{ml}$ MSCGM and cultured for 2 days in 24-well plates. Luciferase activity was measured with BrightGlo (Promega), and Stop \& Glo (Promega) was used for internal control.

\section{siRNA library screening}

The MSCs were infected with Ad-hSF-1 promoter-Luc at $\mathrm{MOI}=5-10$. After $1 \mathrm{~h}$, a human kinome siRNA library (final concentration was $20 \mathrm{nM}$ ) was transfected into the infected cells in 96-well plates (3000 cells/well) by using LipofectAMINE RNAiMax (Invitrogen). Three days after transfection, luciferase activity in each well was measured by Steady-Glo (Promega). Cell growth was also analyzed using CellTiter-Glo (Promega). The similar procedure was used for confirmation studies for siRNA hits coming out of the serial screenings (used $\mathrm{MOI}=10$ of adenovirus vector for reporter construct instead of using $\mathrm{MOI}=5$ to acquire stronger response of luciferase activity).

For the confirmation study with HEK293 cells, especially for the study of PKC- $\delta$ function, cells were grown in 96-well plates at an initial seeding density of $1 \times 10^{4}$ cells/well and cultured for $24 \mathrm{~h}$. pMXs-SIN-hSF-1 promoter-Luc and PKC- $\delta$ cDNAs were co-transfected and cells were analyzed for luciferase expression using Steady-Glo.

\section{Study of function of a PKC- $\delta$ inhibitor, Rottlerin}

The MSCs were infected with Ad-hSF-1 promoter-Luc at $\mathrm{MOI}=10$. After $3 \mathrm{~h}$, cells were re-seeded at $3 \times 10^{3} /$ well in 96-well plates and treated with $0 \cdot 1 \%$ DMSO containing Rottlerin or $0 \cdot 1 \%$ DMSO as a control. Luciferase activity and cell growth were analyzed 4 days after treatment.

\section{DNA-binding assay for SOX15}

Biotin-modified DNA oligonucleotides for wild-type SOXbinding motif (WT-SBM; 5'-GAA ACA CCA ACA AAG AAG GCG AG- $3^{\prime}$ ) and mutant SBM (MT-SBM; 5'-GAA ACA CCT CGA GTC AAG GCG AG-3') were created. Non-modified DNA oligonucleotides for WT-SBM or MT-SBM were also created as specific and non-specific competitors respectively. To perform the DNA-binding assay, the NoShift Transcription Factor Assay Kit (Novagen, San Diego, CA, USA) was used according to the manufacturer's instructions. In brief, biotinylated DNA oligonucleotides of WT-SBM or MT-SBM were mixed with a nuclear extract of SOX15-overexpressing or control pcDNA3.1 introduced into HEK293 cells. In a competition assay, a tenfold excess of competitor (non-modified DNA oligonucleotide of WT-SBM or MT-SBM) was also added. The SOX15-DNA complex was captured on a streptavidin-coated microassay plate. The plate-bound SOX15 protein was then detected with anti-SOX15 antibody (R\&D, Cat\#AF4070) by using the manufacturer's standard procedure. 


\section{Results}

Selection of cell lines for the high-throughput functional screening

We have chosen human bone marrow-derived MSCs and HEK293 cells for the large-scale functional screening.

Human bone marrow-derived MSCs were chosen as an alternative to ovarian MSCs, since it has been reported that MSCs isolated from different tissues share common features (Minguell et al. 2001). Indeed, following ectopic expression of $b N F 5 A 1$ cDNA using an adenovirus vector (Ad-bSF-1), the MSCs produced $17 \beta$-estradiol (Fig. 1a) and progesterone (Fig. 1b). cAMP was added because it is known to induce steroidogenesis in adrenal and gonadal cells (Monté et al. 1998), and indeed production of $17 \beta$-estradiol was additively stimulated by cAMP. In addition to ovarian steroids, ectopic expression of NF5A1 in MSCs resulted in synthesis of DHEA, which is normally produced in adrenal cortex zona reticularis (Fig. 1c) and testosterone (Fig. 1d). DHEA and testosterone production was also stimulated by cAMP. On the other hand, however, endogenous NF5A1 gene expression, which is known to be positively regulated by SF-1 itself (Nomura et al. 1996), was not observed after ectopic expression of SF-1 (data not shown).

MSCs were a suitable cell source for our study; however, the disadvantage of these cells is that its difficulty in cDNA introduction, even siRNA introduction method in these cells was well established in-house, so that they are not practicable for high-content cDNA library screening. To overcome this problem with MSCs, HEK293 cells were chosen for the highcontent cDNA library screening, since this cell line is easily transfected with cDNAs. Moreover, it originated from nephrogenic mesoderm that shares a common origin, intermediate mesoderm, with the genital ridge so that we judged HEK293 to be suitable for the initial gain of function screen with cDNA libraries.

\section{Large-scale gain and loss of function screening}

To gain a comprehensive understanding of NF5A1 gene expression modulators, a human siRNA kinome library and a human cDNA library (MGC cDNA library containing 6344 clones) were screened. The $5 \mathrm{~kb}$ of the NF5A1 promoter (Nomura et al. 1996), including exon 1, intron 1 , and the first part of exon 2 (up to 4 bp upstream of the ATG starting site), was inserted $76 \mathrm{bp}$ upstream of the ATG starting site of a luciferase reporter gene in a pGL4.20 vector (Zhou et al. 2001), then the promoter-reporter constructs were introduced into MSCs and HEK293 cells using adenovirus vector and plasmid transfection respectively. The $517 \mathrm{bp}$ gonadspecific human aromatase promoter II region (from $517 \mathrm{bp}$ upstream of the beginning of exon 2 to one base before exon 2 , which is located $90 \mathrm{bp}$ upstream of the ATG starting site in exon 2; Zhou et al. 2001) was inserted 38 bp upstream of the ATG site of a luciferase reporter gene in pGL4.20 vector and used to validate the function of genes that came up as screening hits (Fig. 2b). For the primary screening, MSCs and
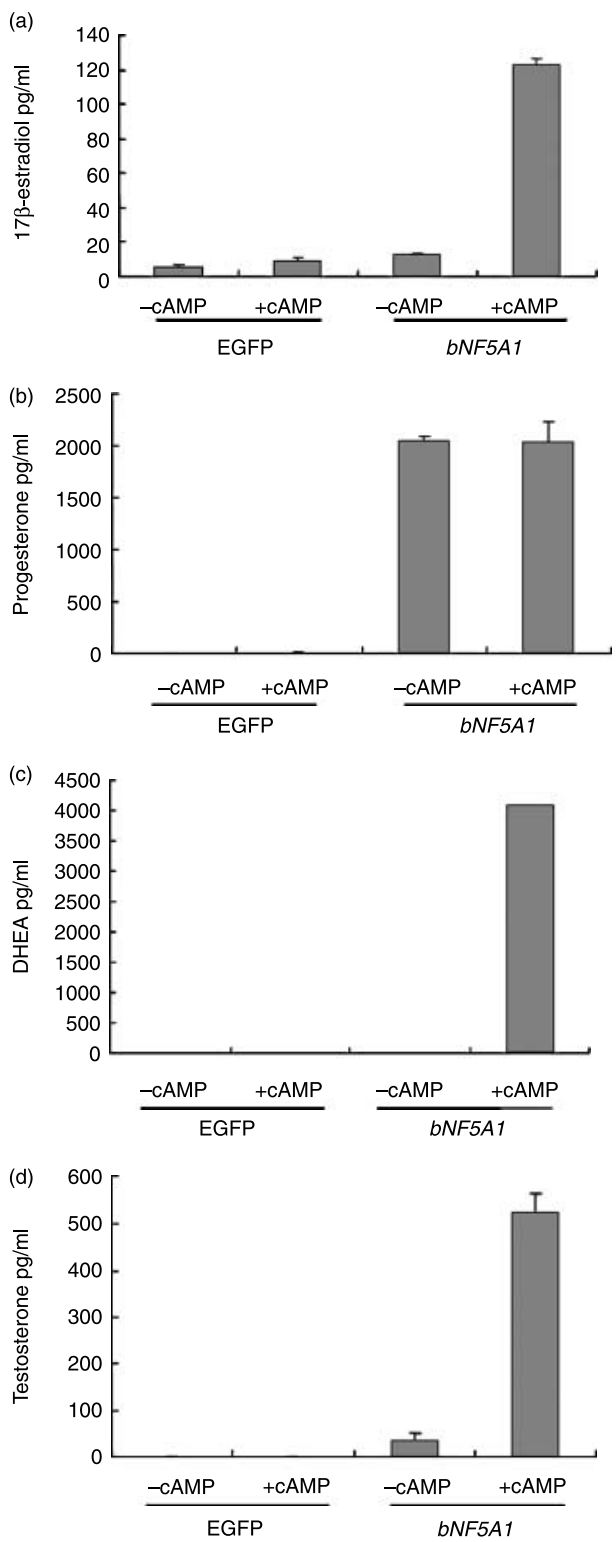

Figure 1 Steroid hormones were produced by the MSCs upon ectopic expression of bovine NF5A1. MSCs were seeded at $5 \times 10^{3}$ cells/well in 96-well plates and infected with adenovirus carrying bovine NF5A1 or EGFP (control) at (a), (b), and (c) multiplicity of infection $(\mathrm{MOI})=2$ or $(\mathrm{d}) \mathrm{MOI}=2 \cdot 5$. The next day, infected MSCs were treated with or without $0.5 \mathrm{mM}$ cAMP and, 6 days later, (a) $17 \beta$-estradiol, (b) progesterone, (c) DHEA, and (e) testosterone production was measured by luminescence immunoassay. Data represent mean \pm s.D. of (a), (b) two, (c) one, and (d) three independent experiments.

HEK293 cells were used for the siRNA and cDNA screening respectively. Four kinases previously unknown to be involved in regulating NF5A1 gene expression were identified by siRNA screening. NF5A1 gene expression was activated in MSCs by siRNA targeted to PKC- $\delta$, erb-B3, RhoGAP (ARHGAP26), and hexokinase 2 (Fig. 2c). The gain of 
(a)

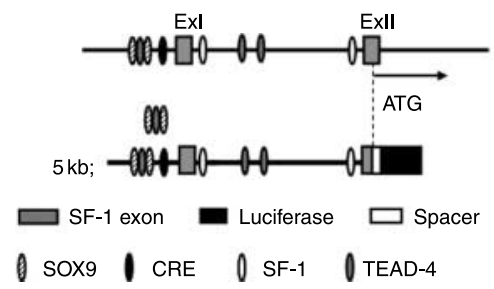

(c)

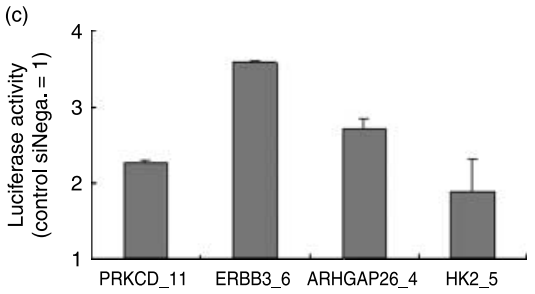

(b)

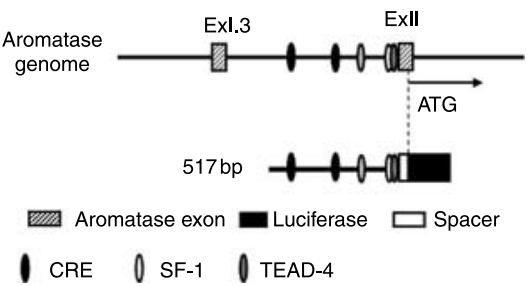

(d)

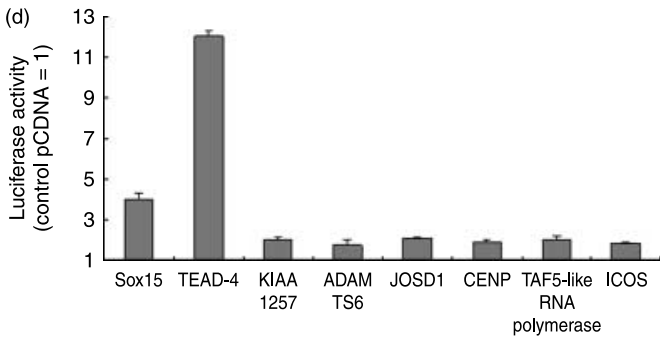

Figure 2 Summary of large-scale functional screening. Structure of reporter construct for (a) human SF-1 promoter (hSF1 promoter-Luc) and (b) human aromatase promoter (hARO promoter-Luc). The transcription start site for each promoter is indicated by an arrow. Gray boxes indicate exons of NF5A1 gene, hatched boxes indicate exons for aromatase gene, and black boxes indicate luciferase cDNA (from pGL4.20 vector). White boxes indicate spacer sequences derived from pGL4.20 and the lengths are 103 bp for the SF-1 reporter construct and 38 bp for the aromatase reporter construct respectively. SOX9binding sites are shown by hatched ovals, CRE-binding sites by black ovals, SF-1-binding sites by open ovals, and TEAD (putative)-binding sites by gray ovals. The luciferase reporter gene is driven by each promoter. (c) Four kinases (PKC- $\delta$ (PRKCD_11), erb-B3 (ERBB3_6), RhoGAP (ARHGAP26_4), and hexokinase 2 (HK-2_5)) were identified as negative regulators of NF5A1 gene expression by human kinome siRNA screening. The MSCs were infected with adenovirus carrying hSF-1 promoter-Luc (Ad-hSF1 promoter-Luc) at $\mathrm{MOI}=10$. After $3 \mathrm{~h}$, infected MSCs were re-seeded at $3 \times 10^{3} /$ well in 96 -well plates and transfected with human kinome siRNA library. (d) SOX15, TEAD-4, KIAA1257, ADAMTS6, JOSD1, CENP, TAF5-like RNA polymerase, and ICOS were identified as positive regulators of NF5A1 gene expression by human cDNA library screening. HEK293 cells were seeded at $6 \times 10^{3} /$ well in 96 -well plates and transiently transfected with $10 \mathrm{ng}$ hSF-1 promoter-Luc together with 25 ng human cDNA. The luciferase activity was measured 3-4 days after transfections. Data were normalized and represent mean \pm s.D. of two to three independent experiments.

function screening by introducing cDNAs revealed that SOX15, TEAD-4, KIAA1257 (a gene of unknown function), ADAM metallopeptidase with thrombospondin type 1 motif 6 (ADAMTS6), JOSD1, CENP, TAF5, and ICOS activate NF5A1 gene expression in HEK293 cells (Fig. 2d).

\section{Identification of the PKC- $\delta / e r b-B 3$ module}

Among the four kinase siRNAs identified in this study, the PKC- $\delta$ and erb-B3 siRNA showed significant stimulation of NF5A1 gene expression in MSCs. As the MSCs used in this study express five PKC subtypes, PKC- $\alpha, \mathrm{PKC}-\delta, \mathrm{PKC}-\varepsilon$, PKC- $\eta$, and PKC-ı (data not shown), we first studied the subtype specificity (Dempsey et al. 2000, Woods \& Johnson 2007). Although multiple siRNAs for PKC- $\delta$ activated the SF-1 promoter reporter system in the MSCs, none of the siRNAs for PKC- $\alpha$, PKC- $\varepsilon$, PKC- $\eta$, or PKC-ı activated the SF-1 promoter reporter system (Fig. 3a). In accordance with the siRNA studies, ectopic expression of PKC- $\delta$ cDNA by transfection dose dependently inhibited the SF-1 promoter reporter system in HEK293 cells (Fig. 3b). In addition, Rottlerin, an inhibitor of $\mathrm{PKC}-\delta$, dose dependently activated the SF-1 reporter promoter in MSCs (Fig. 3c).

Next, we examined the relationship between PKC- $\delta$ and erb-B3. It has been shown that erb-B3-binding protein (Ebp1; Liu et al. 2007), a member of the PA2G4 family, binds to nuclear Akt by phosphorylation mediated by $\mathrm{PKC}-\delta$. Consistent with this finding, siRNA against Ebp1 increased SF-1 promoter reporter activity (Fig. 3d). These results indicate that erb-B3, PKC- $\delta$, and Ebp1 make up an inhibitory signaling module for NF5A1 gene expression in MSCs.

\section{Effect of the transcription factors SOX 15 and TEAD-4 on NF5A1 gene expression}

Among the eight cDNAs that activated the SF-1 promoter in HEK293 cells, we focused our studies to two transcription factors, SOX15 (Hiraoka et al. 1998) and TEAD-4 (Thompson et al. 2003). These two factors, as well as NF5A1, activated the SF-1 promoter reporter system in MSCs and HEK293 cells (Fig. 4a and b). In addition, SOX15, TEAD-4, and $b N F 5 A 1$ activated the aromatase promoter (Fig. 4c). The bSF-1 expression vector 

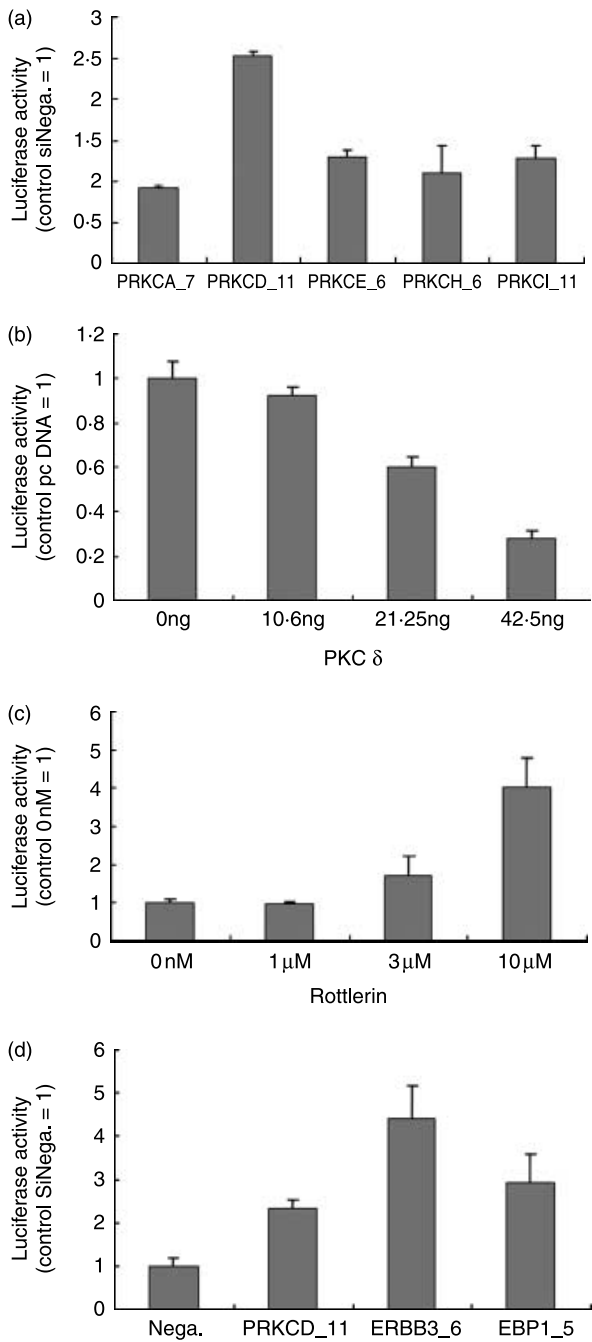

Figure 3 Effect of PKC- $\delta$, Ebp1, and erb-B3 on SF-1 promoter inhibition. (a) From the PKC family, PKC- $\delta$ specifically regulated SF-1 promoter activity. (b) Ectopic expression of PKC- $\delta$ inhibited SF-1 promoter activity in a dose-dependent manner. (c) Rottlerin, a PKC- $\delta$ inhibitor, enhanced SF-1 promoter activity in a concentrationdependent manner. (d) Knockdown of erb-B3-binding protein Ebp1 enhanced SF-1 promoter activity. For (a), (c), and (d), the MSCs were infected with Ad-hSF-1 promoter-Luc at (a), (d) MOI=5, or (c) $\mathrm{MOI}=10$. After $3 \mathrm{~h}$, infected MSCs were re-seeded at $3 \times 10^{3} /$ well in 96-well plates and transfected with siRNA or treated with Rottlerin. The luciferase activity was analyzed 4 days after transfection or treatment. Data were normalized and represent mean \pm s.D. of three independent experiments. For (b), HEK293 were seeded at $1 \times 10^{4} /$ well in 96-well plates and transiently transfected with 17 ng pMXs-SIN-hSF-1 promoter-Luc together with $0,10 \cdot 6$, $21 \cdot 25$, and $42 \cdot 5$ ng PKC- $\delta$ expression vector or control pcDNA3. 1 plasmid. The luciferase activity was analyzed 3 days after transfection. Data were normalized and represent mean \pm s.D. of at least six independent experiments. PRKCA_7, PKC- $\alpha$; PRKCD_11,

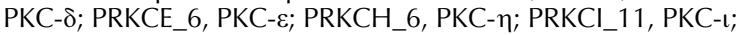
ERBB3_6, erb-B3; EBP1_5, Ebp1/PA2G4. was used as a positive control because the SF-1 and aromatase promoter reporter systems used in this study contain SF-1-binding sites (Fig. 2a and b). SOX15binding sites on the SF-1 and aromatase promoters have not previously been studied. However, Shen \& Ingraham (2002) described regulation of NF5A1 expression by SOX proteins, particularly $\mathrm{SOX} 3, \mathrm{SOX} 8$, and $\mathrm{SOX} 9$, and they showed that all of these SOX proteins were able to bind to the proximal SOX-binding site within the SF-1 promoter. Thus, we expected that SOX15 is also binding to the same site. To identify whether SOX15 binds directly to the SOX-binding site in the SF-1 proximal promoter region, we performed DNA-binding studies using a DNA-binding ELISA (Fig. 4d). SOX15 specifically bound to the SOX responsive element on the SF-1 promoter, and this binding competed with that of an oligonucleotide with the SOX-binding sequence (Fig. 4d). This result indicates that SOX15 directly binds to the SOX-binding site on the SF-1 promoter. We could not identify a SOX15-binding site on the aromatase promoter, indicating that the effects of SOX15 in activating the aromatase reporter system may be via indirect pathways.

Next, we examined the subtype specificity for the SOX family using the SF-1 reporter system in HEK293 cells. The MGC cDNA library that was used for our gain of function study included five human $S O X$ family transcription factors (Kawabe et al. 1999), SOX8, SOX9, SOX15, SOX18, and SOX30, and these SOX proteins were used for this analysis (Fig. 4e). Although all five factors activated the SF-1 reporter system, SOX15 and SOX30 showed significant activation.

cAMP response element-binding protein (CREB), is a transcription factor that activates the expression of steroidogenic genes, such as STAR and P450aromatase (Mendelson \& Kamat 2007). Recently, it has been reported that CREB and SF-1 proteins recruit a complex of transcriptional coactivators to target gene promoters (Weck \& Mayo 2006). It is also known that one of the SOX family proteins, SOX9, and CREB bind to regulatory regions on the SF-1 promoter to induce gene expression (Val et al. 2003). Therefore, we next studied whether SOX15 also had a synergistic effect on the SF-1 reporter system together with CREB (Fig. 4f). Ectopic expression of CREB clearly activated the SF-1 promoter reporter system and, like SOX9, SOX15 and CREB had a synergistic effect on the SF-1 promoter.

As mentioned above, TEAD-4 forced expression also activated SF-1 and aromatase reporter gene expression. Therefore, we analyzed promoter regions in both the SF-1 and aromatase genes and found three putative TEAD-4-binding sites in the SF-1 promoter region we used for the reporter system and one in the aromatase promoter region of the reporter construct (Jacquemin et al. 1996, Anbanandam et al. 2006). Based on this finding and on the published information, we suggest that TEAD-4 could also be an important regulator of SF-1 and aromatase gene expression. 


\section{Discussion}

HRT is widely used for patients suffering from deficiency in production of a number of steroid hormones, glucocorticoids (adrenal failure), or estrogens (menopausal disorders). However, a limitation of current HRT is the difficulty in dose
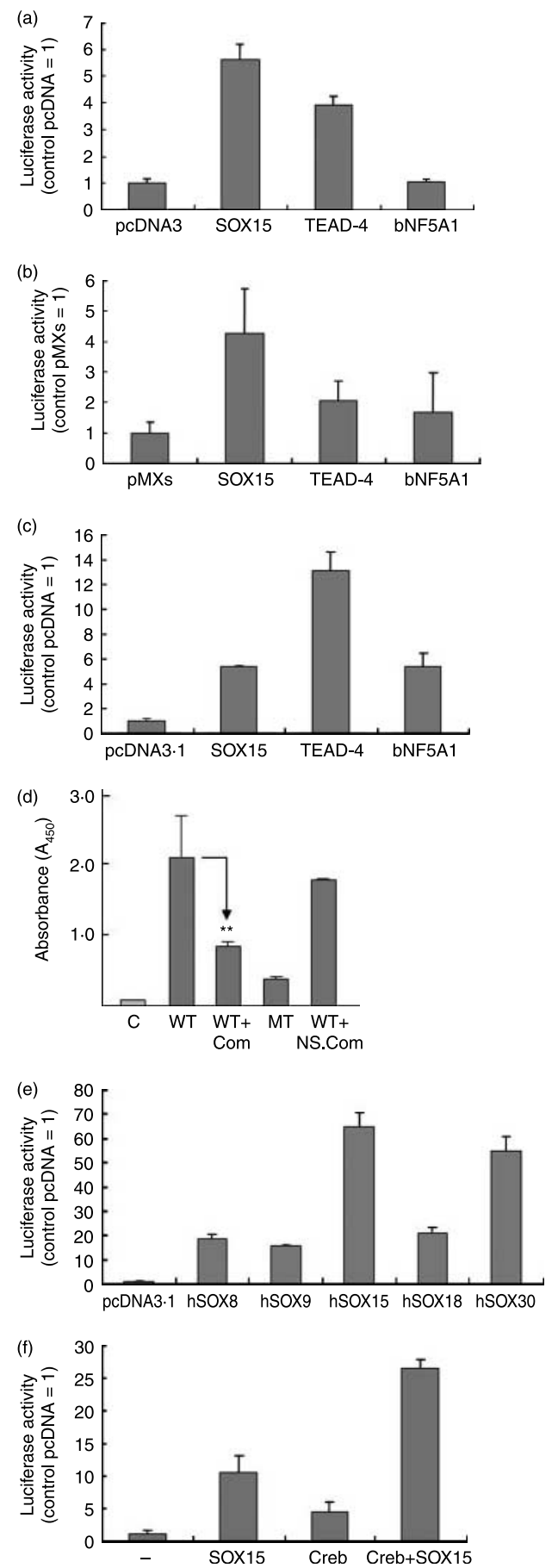

www.endocrinology-journals.org optimization. For this reason, some groups are now trying to develop a method to transplant ex vivo-generated steroidogenic cells to allow control of hormone concentrations in blood by the patients' own hypothalamus-anterior pituitary axis (Gondo et al. 2004, Yazawa et al. 2006, Tanaka et al. 2007).

Alternatively, as we suggest here, it might be possible to regulate de novo NF5A1 expression pharmacologically by targeting molecules involved in NF5A1 gene expression regulation.

Although genome-wide functional screening will pick up a large amount of non-druggable targets, in silico pathway analysis will be able to identify the druggable molecules directly or indirectly connected to these targets. Inhibitors for these druggable molecules also would not be sufficient to make pharmacological effect separately. However, the combination of these 'weak' inhibitors would be able to make pharmacological effect that is defined as 'long-tail' drugs (Kitano 2007).

In this study, a large-scale functional screening method allowed us to identify four kinase that were previously not known to be involved in regulating NF5A1 gene expression. In addition, we identified that PKC- $\delta$ and erb-B3 together with Ebp1 make up an inhibitory module for NF5A1 gene expression. It has been shown that EGF-like growth factors such as amphiregulin, epiregulin, and $\beta$-cellulin mediate the action of LH in the ovulatory follicle (Park et al. 2004). In addition, EGF-like growth factors are considered as negative regulators of FSH-related steroidogenesis (Park et al. 2004). Our study indicates that EGF-like growth factor-mediated inhibition of steroidogenesis may be mediated through the repression of $N F 5 A 1$ gene expression.

In the gain of function screening, eight $\mathrm{CDNAs}$ that were previously not known to activate NF5A1 gene expression were identified. Four of them, SOX15, TEAD-4, CENP, and TAF5like RNA polymerase, are nuclear proteins. In addition, ADAMTS6, JOSD1, ICOS, and KIAA1257 were identified. In this study, we focused our studies to two transcription factors, SOX15 and TEAD-4. Although it is well known that SOX9 regulates $N F 5 A 1$ gene expression, we have identified that

Figure 4 The transcription factors SOX and TEAD-4 significantly increased the promoter activity for both SF-1 and aromatase. (a and b) Both SOX 15 and TEAD-4 efficiently enhanced SF-1 promoter activity in (a) HEK293 cells and (b) the MSCs. (c) Both SOX15 and TEAD-4 also increased aromatase promoter activity by using pMXs-SIN-hAro promoter-Luc. (d) SOX15 specifically binds to the SOX-binding site on the SF-1 promoter region. The core SOX-binding site (AACAAAG) was used in a NoShift competitor assay to measure the DNA-binding activity of hSOX15. WT-SBM (WT) or MT-SBM (MT) was used in this assay in the presence or absence of a SBM-specific competitor (Com) or a non-specific competitor (NS.Com). ${ }^{* * P}<0 \cdot 05$ compared with WT. (e) Other SOX family members also increased SF-1 promoter activity. (f) Synergistic effects of SOX15 and CREB on SF-1 promoter activity. HEK293 cells or MSCs were transfected with the SF-1 or aromatase reporter gene together with the indicated expression plasmids. The expression plasmid of bNF5A1 was used as a positive control. After 2 days, cell extracts were prepared and measured for luciferase activity. Data were normalized and represent mean \pm s.D. of at least three independent experiments. 
SOX15 can also stimulate NF5A1 gene expression significantly. The NF5A1 gene expression profile is still controversial. For example, NF5A1 expression was reported to be upregulated in the developing male gonad (Sarraj et al. 2003); however, SOX15 protein was only found within the cytoplasm of $13.5 \mathrm{dpc}$ Sertoli cells. In the same study, Sarraj et al. showed that SOX15 was not expressed in the adult ovary. In contrast, Assou et al. (2006) found that SOX15 was overexpressed in oocytes in their study of the mechanisms regulating human oocyte maturation. Although our result is consistent with the results and hypothesis of Shen \& Ingraham (2002) that SOX15 is expressed in adult Leydig cells and is involved in NF5A1 expression during Leydig cell differentiation, the role of SOX15 in the theca/granulosa cell differentiation is not yet to be concluded without the evidence that SOX15 is expressed in the somatic stem cells in ovary.

In the present study, we found that not only SOX15 but also SOX 30 is a strong regulator of the SF-1 promoter. Osaki et al. (1999) found SOX30 to be exclusively expressed in adult testis and showed an exclusive germ cell-specific expression indicating that $S O X 30$ might be involved in spermatogenesis. Although, in the same study, they reported that SOX30 was not detected in adult ovary by northern blot experiments, Assou et al. reported expression of SOX30 in oocytes. SOX30 might be an important molecule for germ stem cell differentiation. Like SOX15, SOX30 may be a regulator of steroidogenic cell differentiation in adult stem cells in the testis or ovaries. However, as mentioned above, in 2002, Shen \& Ingraham studied the functions of $S O X 3, S O X 8$, and $S O X 9$ in regulation of NF5A1 expression, and showed that even though all three of them bind to the proximal SOX-binding site within the SF-1 promoter, only SOX9 is able to transactivate NF5A1 gene expression so that each SOX protein required appropriate factors as its partners to activate NF5A1 to achieve tissue-specific gene expression. Therefore, further study will be required to understand about the function of SOX 15 and SOX 30 in various types of steroidogenic tissues.

In addition, we have identified that TEAD-4 significantly upregulated the SF-1 and aromatase promoters and putative TEAD-4-binding sites exist on the SF-1 promoter and the aromatase promoter. It has been reported that TEAD-4 is expressed in the ovarian epithelial cancer cell line OVCAR-3 and a placenta cell line that expresses NF5A1 and aromatase (JEG-3; Harris \& Mellon 1998). Thus, TEAD-4 might have a physiological role in regulating NF5A1 expression. TEAD-4 is a member of the transcription enhancer factor family that shares a common TEA-DNA-binding domain. Four transcription factors (TEAD-1, TEAD-2, TEAD-3, and TEAD-4) bind to the same DNA sequence. One of these transcription factors may have a physiological role in SF-1 promoter activation.

ADAMTS6 is a secreted protein that is a member of the extracellular metalloproteinase family containing disintegrinlike domains (ADAMs). The function of ADAMTS6 is still not well understood; however, it is known that extracellular matrix remodeling in ovaries is very important in folliculogenesis and ovulation (Berkholtz et al. 2006) and, moreover, one of the ADAMTS family members, ADAMTS-1, is necessary for structural remodeling during ovarian follicle growth (Brown et al. 2006). Therefore, it is possible that secreted ADAMTS6 may also affect steroidogenesis in follicular cells.

ICOS is a member of the CD28 family of costimulatory molecules expressed primarily on activated human $\mathrm{T}$ cells (van Berkel \& Oosterwegel 2006). ICOS activation of SF-1 reporter gene expression might occur through the action of ICOS ligand on HEK293 cells, and downstream signaling of ICOS ligand may activate NF5A1 expression. This appears plausible as follicles are surrounded by lymphocytes and it is known that the immune system contributes to the regulation of gonadal function (Bukulmez \& Arici 2000). Since our gene identification was based on functional screening, homologs of some of the identified genes may actually function in the steroid-producing cells. Further studies would be expected for the expression and function of all identified target genes on NF5A1 gene regulations across various endocrine tissues. Expression of identified genes might be restricted to stem/ progenitor/blast cells in tissues, which make up a very small part of the total cell population, or might be more widely expressed. Moreover, time course of their expression may also play a major role for $N F 5 A 1$ gene regulation. These points will also need to be clarified in the future study.

These results provide new insights into the molecular mechanisms of NF5A1 gene expression as well as molecular targets for pharmacological intervention to treat diseases caused by a reduction of estrogen production in the ovary, such as menopausal disorders.

\section{Declaration of interest}

The authors declare that there is no conflict of interest that would prejudice its impartiality.

\section{Funding}

The work was supported by Bayer-Schering Pharma.

\section{Acknowledgements}

We thank M Adachi, H Masaki, K Kominami, H Migita, and S Takahashi for advice and technical support.

\section{References}

Anbanandam A, Albarado DC, Nguyen CT, Halder G, Gao X \& Veeraraghavan S 2006 Insights into transcription enhancer factor 1 (TEF-1) activity from the solution structure of the TEA domain. PNAS 103 17225-17230.

Assou S, Anahory T, Pantesco V, Le Carrour T, Pellestor F, Klein B, Reyftmann L, Dechaud H, De Vos J \& Hamamah S 2006 The human cumulus-oocyte complex gene-expression profile. Human Reproduction 21 1705-1719.

van Berkel ME \& Oosterwegel MA 2006 CD28 and ICOS: similar or separate costimulators of T cells? Immunology Letters 105 115-122.

Berkholtz CB, Shea LD \& Woodruff TK 2006 Extracellular matrix functions in follicle maturation. Seminars in Reproductive Medicine 24 262-269.

www.endocrinology-journals.org 
Broekmans FJ, Knauff EA, te Velde ER, Macklon NS \& Fauser BC 2007 Female reproductive ageing: current knowledge and future trends. Trends in Endocrinology and Metabolism 18 58-65.

Brown HM, Dunning KR, Robker RL, Pritchard M \& Russell DL 2006 Requirement for ADAMTS-1 in extracellular matrix remodeling during ovarian folliculogenesis and lymphangiogenesis. Developmental Biology $\mathbf{3 0 0}$ 699-709.

Bukulmez O \& Arici A 2000 Leukocytes in ovarian function. Human Reproduction Update 6 1-15.

Chan EY, Kir S \& Tooze SA 2007 siRNA screening of the kinome identifies ULK1 as a multidomain modulator of autophagy. Journal of Biological Chemistry 282 25464-25474.

Cirigliano M 2007 Bioidentical hormone therapy: a review of the evidence. Journal of Women's Health 16 600-631.

Clyne CD, Speed CJ, Zhou J \& Simpson ER 2002 Liver receptor homologue1 (LRH-1) regulates expression of aromatase in preadipocytes. Journal of Biological Chemistry 277 20591-20597.

Crawford PA, Sadovsky Y \& Milbrandt J 1997 Nuclear receptor steroidogenic factor 1 directs embryonic stem cells toward the steroidogenic lineage. Molecular and Cellular Biology 17 3997-4006.

Dempsey EC, Newton AC, Mochly-Rosen D, Fields AP, Reyland ME, Insel PA \& Messing RO 2000 Protein kinase C isozymes and the regulation of diverse cell responses. American Journal of Physiology. Lung Cellular and Molecular Physiology 279 L429-L438.

Gondo S, Yanase T, Okabe T, Tanaka T, Morinaga H, Nomura M, Goto K \& Nawata H 2004 NF5A1/Ad4BP transforms primary long-term cultured bone marrow cells into ACTH-responsive steroidogenic cells. Genes to Cells 9 1239-1247.

Harris AN \& Mellon PL 1998 The basic helix-loop-helix, leucine zipper transcription factor, USF (upstream stimulatory factor), is a key regulator of NF5A1 (steroidogenic factor-1) gene expression in pituitary gonadotrope and steroidogenic cells. Molecular Endocrinology 12 714-726.

Hiraoka Y, Ogawa M, Sakai Y, Taniguchi K, Fujii T, Umezawa A, Hata J \& Aiso S 1998 Isolation and expression of a human SRY-related cDNA hSOX20. Biochimica et Biophysica Acta 1396 132-137.

Honda A, Hirose M, Hara K, Matoba S, Inoue K, Miki H, Hiura H, KanatsuShinohara M, Kanai Y, Kono T et al. 2007 Isolation, characterization, and in vitro and in vivo differentiation of putative thecal stem cells. PNAS 104 12389-12394.

Jacquemin P, Hwang JJ, Martial JA, Dollé P \& Davidson I 1996 A novel family of developmentally regulated mammalian transcription factors containing the TEA/ATTS DNA binding domain. Journal of Biological Chemistry 271 21775-21785.

Kawabe K, Shikayama T, Tsuboi H, Oka S, Oba K, Yanase T, Nawata H \& Morohashi K 1999 Dax-1 as one of the target genes of Ad4BP/NF5A1. Molecular Endocrinology 13 1267-1284.

Kitano H 2007 A robustness-based approach to systems-oriented drug design. Nature Reviews. Drug Discovery 6 202-210.

Liu Z, Liu X, Nakayama KI, Nakayama K \& Ye K 2007 Protein kinase C-delta phosphorylates Ebp1 and prevents its proteolytic degradation, enhancing cell survival. Journal of Neurochemistry 100 1278-1288.

Magoffin DA 2005 Ovarian theca cell. International Journal of Biochemistry and Cell Biology 37 1344-1349.

Mendelson CR \& Kamat A 2007 Mechanisms in the regulation of aromatase in developing ovary and placenta. Journal of Steroid Biochemistry and Molecular Biology 106 62-70.

Minguell JJ, Erices A \& Conget P 2001 Mesenchymal stem cells. Experimental Biology and Medicine 226 507-520.

Monté D, DeWitte F \& Hum DW 1998 Regulation of the human P450scc gene by steroidogenic factor 1 is mediated by $\mathrm{CBP} / \mathrm{p} 300$. Journal of Biological Chemistry 273 4585-4591.

Morohashi K, Honda S, Inomata Y, Handa H \& Omura T 1992 A common trans-acting factor, Ad4-binding protein, to the promoters of steroidogenic P-450s. Journal of Biological Chemistry 267 17913-17919.

Nomura M, Nawata H \& Morohashi K 1996 Autoregulatory loop in the regulation of the mammalian ftz-f1 gene. Journal of Biological Chemistry 271 8243-8249.
Osaki E, Nishina Y, Inazawa J, Copeland NG, Gilbert DJ, Jenkins NA, Ohsugi M, Tezuka T, Yoshida M \& Semba K 1999 Identification of a novel Sry-related gene and its germ cell specific expression. Nucleic Acids Research 27 2503-2510.

Palter SF, Tavares AB, Hourvitz A, Veldhuis JD \& Adashi EY 2001 Are estrogens of import to primate/human ovarian folliculogenesis? Endocrine Reviews 22 389-424.

Park JY, Su YQ, Ariga M, Law E, Jin SL \& Conti M 2004 EGF-like growth factors as mediators of LH action in the ovulatory follicle. Science 303 682-684.

Rossouw JE, Anderson GL, Prentice RL, LaCroix AZ, Kooperberg C, Stefanick ML, Jackson RD, Beresford SA, Howard BV, Johnson KC et al. 2002 Risks and benefits of estrogen plus progestin in healthy postmenopausal women: principal results From the Women's Health Initiative randomized controlled trial. Journal of the American Medical Association 288 321-333.

Sarraj MA, Wilmore HP, McClive PJ \& Sinclair AH 2003 SOX15 is up regulated in the embryonic mouse testis. Gene Expression Patterns 3 413-417.

Saxena D, Escamilla-Hernandez R, Little-Ihrig L \& Zeleznik AJ 2007 Liver receptor homolog-1 and steroidogenic factor-1 have similar actions on rat granulosa cell steroidogenesis. Endocrinology 148 726-734.

Scherrer SP, Rice DA \& Heckert LL 2002 Expression of steroidogenic factor 1 in the testis requires an interactive array of elements within its proximal promoter. Biology of Reproduction 67 1509-1521.

Shen JH \& Ingraham HA 2002 Regulation of the orphan nuclear receptor steroidogenic factor 1 by SOX proteins. Molecular Endocrinology 16 529-540.

Simpson ER \& Davis SR 2001 Minireview: aromatase and the regulation of estrogen biosynthesis - some new perspectives. Endocrinology 142 4589-4594.

Tanaka T, Gondo S, Okabe T, Ohe K, Shirohzu H, Morinaga H, Nomura M, Tani K, Takayanagi R, Nawata H et al. 2007 Steroidogenic factor 1/adrenal 4 binding protein transforms human bone marrow mesenchymal cells into steroidogenic cells. Journal of Molecular Endocrinology 39 343-350.

Thompson M, Andrade VA, Andrade SJ, Pusl T, Ortega JM, Goes AM \& Leite MF 2003 Inhibition of the TEF/TEAD transcription factor activity by nuclear calcium and distinct kinase pathways. Biochemical and Biophysical Research Communications 301 267-274.

Tyner JW, Walters DK, Willis SG, Luttropp M, Oost J, Loriaux M, Erickson H, Corbin AS, O'Hare T, Heinrich MC et al. 2008 RNAi screening of the tyrosine kinome identifies therapeutic targets in acute myeloid leukemia. Blood 111 2238-2245.

Val P, Lefrançois-Martinez AM, Veyssière G \& Martinez A 2003 NF5A1 a key player in the development and differentiation of steroidogenic tissues. Nuclear Receptor 18.

Vanderhyden B 2002 Molecular basis of ovarian development and function. Frontiers in Bioscience 7 d2006-d2022.

Weck J \& Mayo KE 2006 Switching of NR5A proteins associated with the inhibin alpha-subunit gene promoter after activation of the gene in granulosa cells. Molecular Endocrinology 20 1090-1103.

Woods DC \& Johnson AL 2007 Protein kinase C activity mediates LH-induced ErbB/Erk signaling in differentiated hen granulosa cells. Reproduction 133 733-741.

Yazawa T, Mizutani T, Yamada K, Kawata H, Sekiguchi T, Yoshino M, Kajitani T, Shou Z, Umezawa A \& Miyamoto K 2006 Differentiation of adult stem cells derived from bone marrow stroma into Leydig or adrenocortical cells. Endocrinology 147 4104-4111.

Zhou J, Gurates B, Yang S, Sebastian S \& Bulun SE 2001 Malignant breast epithelial cells stimulate aromatase expression via Promoter II in human adipose fibroblasts: an epithelial-stromal interaction in breast tumors mediated by CCAAT/enhancer binding protein. Cancer Research 61 2328-2334.

de Ziegler D, Fraisse T, de Candolle G, Vulliemoz N, Bellavia M \& Colamaria S 2007 Outlook: roles of FSH and LH during the follicular phase: insight into natural cycle IVF. Reproductive Biomedicine Online 15 507-513.

Received in final form 11 June 2008

Accepted 25 June 2008

Made available online as an Accepted Preprint 25 June 2008 